\title{
Aminoglycoside antibiotics for NIH category II chronic bacterial prostatitis: A single-cohort study with one-year follow-up
}

\author{
VITTORIO MAGRI $^{1 *}$, EMANUELE MONTANARI $^{2 *}$, EMANUELA MARRAS $^{3}$ and GIANPAOLO PERLETTI ${ }^{3,4}$ \\ ${ }^{1}$ Urology Secondary Care Clinic, Azienda Socio-Sanitaria Territoriale-Nord, I-20132 Milan; \\ ${ }^{2}$ Urology Complex Unit, Ca' Granda Foundation, IRCCS Ospedale Maggiore Policlinico di Milano, I-20122 Milan; \\ ${ }^{3}$ Department of Biotechnology and Life Sciences, Section of Medical and Surgical Sciences, \\ University of Insubria, I-21052 Busto Arsizio, Italy; ${ }^{4}$ Department of Basic Medical Sciences, \\ Faculty of Medicine and Health Sciences, Ghent University, B-9000 Ghent, Belgium
}

Received April 14, 2016; Accepted July 21, 2016

DOI: 10.3892/etm.2016.3631

\begin{abstract}
Although fluoroquinolones are first-line agents for the treatment of National Institutes of Health (NIH) category II chronic bacterial prostatitis (CBP), therapy with these agents is not always feasible due to the increasing worldwide resistance of causative uropathogens. New therapeutic options are urgently required, as drugs such as $\beta$-lactam antibiotics distribute poorly to prostatic sites of infection and trimethoprim therapy is often unfeasible due to high resistance rates. The present study aimed to analyze the efficacy of aminoglycosides, administered to a cohort of 78 patients affected by fluoroquinolone-resistant CBP, or excluded from fluoroquinolone therapy due to various contraindications. Patients received netilmicin $(4.5 \mathrm{mg} / \mathrm{kg}$, once-daily, intramuscular), combined or not with a $\beta$-lactam antibiotic, for 4 weeks. Follow-up visits were scheduled 6 and 12 months after the end of treatment. Fifty-five out of 70 patients (78.6\%) showed eradication of the causative pathogen, and a significant reduction of the NIH-Chronic Prostatitis Symptom Index (NIH-CPSI) total score from a baseline median value of 21 to 14 at the end of therapy, and to 9 and 8 at 6-month and 12-month follow-up assessments, respectively. The pain, voiding and quality of life subdomains of the NIH-CPSI decreased accordingly. In 15 patients showing persistence of infection, NIH-CPSI total and subdomain scores did not decrease at the end of therapy. Additional clinical parameters, such as the urinary peak
\end{abstract}

Correspondence to: Professor Gianpaolo Perletti, Department of Biotechnology and Life Sciences, University of Insubria, 12 Via Manara, I-21052 Busto Arsizio, Italy

E-mail: gianpaolo.perletti@uninsubria.it

*Contributed equally

Key words: prostatitis, chronic bacterial prostatitis, chronic pelvic pain syndrome, Meares and Stamey test, fluoroquinolones, aminoglycosides, amikacin, gentamicin, netilmicin, National Institutes of Health-chronic prostatitis symptom index flow rate, percentage voided bladder, serum prostate-specific antigen concentration, International Prostate Symptom Score and prostate volume improved significantly only in the group of patients in which the infection was eradicated. Therapy was well tolerated, and genetic testing for deafness-predisposing mitochondrial mutations allowed safer administration of aminoglycosides. These results suggest that aminoglycosides may become a therapeutic alternative for the treatment of CBP. These findings should be further validated in a randomized-controlled setting.

\section{Introduction}

There is mounting evidence that a history of clinical chronic prostatitis can be a risk factor for prostate cancer (1-7). As a consequence, aggressive therapy, aimed at eradicating or neutralizing the etiological agents of bacterial or abacterial forms of chronic prostatitis, is of great importance for the prompt resolution of the severe symptoms of these conditions, but may also have implications in cancer prevention.

National Institutes of Health (NIH) category II chronic bacterial prostatitis (CBP) and category III chronic prostatitis/chronic pelvic pain syndrome are the two main chronic symptomatic conditions of the prostate (8). While the latter is often treated with a variety of agents in an attempt to target its manifold and often uncertain etiological determinants, the former is usually managed with antibacterial agents, administered at high doses and for extended periods of time (weeks, or in some cases, months).

Fluoroquinolones such as ciprofloxacin or levofloxacin have been first-line agents for the treatment of CBP for many years, due to their broad antibacterial spectrum and efficient distribution to the prostate tissue and glandular ducts (9). However, fluoroquinolone-based therapy is not always feasible, due to specific contraindications (for example, a history of tendonitis or long QT syndrome) or to resistance of causative uropathogens to these agents $(10,11)$. In this regard, the resistance rates of Enterobacteriaceae and other pathogens causing community-acquired or healthcare-associated urogenital infections are increasing markedly. They exceed $50 \%$ in many parts of the world, particularly in Asia, but 
similar figures have been reported in other continents. For example, fluoroquinolone resistance rates in Europe and North America range from $\sim 10 \%$ in rural areas to $>30 \%$ in established sexual networks (12).

Although national and international implementation of targeted stewardship may eventually decrease the deleterious impact of bacterial chemoresistance (13), new therapeutic options are urgently required as alternatives to fluoroquinolones. Regretfully, the armamentarium for the outpatient treatment of CBP appears to be quite limited, as most $\beta$-lactams distribute very poorly to the prostatic tissue, and trimethoprim therapy is in the majority of cases unfeasible, since worldwide resistance rates to cotrimoxazole are equivalent to or higher than those observed for fluoroquinolones, and cases of fluoroquinolone-cotrimoxazole cross-resistance are frequently reported (14).

Aminoglycosides are among the recommended agents for the treatment of category I acute bacterial prostatitis (ABP) (15). A retrospective multi-center analysis conducted in 2008 showed that an aminoglycoside is administered in $80 \%$ of cases of ABP (16). However, to the best of our knowledge, only case reports or small case series describing the successful administration of these agents to patients affected by $\mathrm{CBP}$ are available (17). Hence, new evidence is required to support the usage of aminoglycosides for the treatment of CBP. The present study aimed to analyze the efficacy and safety of systemic administration of aminoglycoside antibiotics to a cohort of 78 patients affected by fluoroquinolone-resistant $\mathrm{CBP}$, or excluded from fluoroquinolone therapy due to various contraindications.

\section{Patients and methods}

The Ethics Committee of the Principal Investigator's Hospital was notified of the present study, according to Italian bylaws. This was a subordinate study of a larger observational investigation, recorded in the Italian Medicines Agency AIFA clinical trial register (no. 276). For an observational retrospective study, approval is not required, and a simple notification accompanying the study protocol is sufficient (Determinazione AIFA 20/3/2008, GU 76, 31/3/2008). Patients provided written consent for processing and anonymous publication of their clinical data. This manuscript was prepared in adherence to the Strengthening the Reporting of Observational Studies in Epidemiology Statement (STROBE) guidelines for good reporting of observational studies (18).

Inclusion criteria. The present study was based on the retrospective analysis of a database of $>1,700$ patients with chronic prostatitis who were diagnosed and treated on an outpatient basis in Milan, Italy. Patients consecutively diagnosed with CBP from year 2001 onwards were considered.

Patients between 20 and 65 years of age were included in this study in the presence of a clinical indication for treatment with aminoglycoside antibiotics of category II CBP, diagnosed according to NIH criteria, defined in 1995 at the National Institute of Diabetes and Digestive and Kidney Diseases Chronic Prostatitis Workshop (Bethesda, MD, USA).

Patients were excluded if they presented any of the following conditions: Category I acute bacterial prostatitis, therapy with antibacterial agents or any medication effective at the prostatic level within a 90 -day period prior to aminoglycoside treatment, renal/hepatic/cardiac insufficiency, elevated creatinine plasma levels, a family history or audiometric evidence of hearing impairment, indwelling catheters, cystostomy or ureterostomy, previous prostatic surgery or radiotherapy, incomplete compliance with antibacterial therapy assessed by interviewing patients at test-of-cure visits, any condition that might represent a major confounder in the evaluation of the patients with the NIH Chronic Prostatitis Symptom Index (NIH-CPSI) for example, chronic consumption of drugs such as antidepressants or tramadol.

Diagnostic procedures and symptom evaluation. CBP was diagnosed as previously described in detail (19). Briefly, the patient evaluation was based on careful collection of clinical history, a urological visit including a digitorectal examination, transrectal ultrasound, urine flowmetry [assessment of peak urinary flow rate $\left.\left(\mathrm{Q}_{\max }\right)\right]$, and on microbiological analysis and antibiogram of lower urinary tract specimens obtained with the Meares \& Stamey '4-glass' test (20). The total ejaculate was collected thereafter, and analyzed for pathogens and leukocytes, as previously described (21).

For the diagnosis of prostatic bacterial infection, colony counts in prostatic specimens (expressed prostatic secretions or post-massage urine) were required to be $\geq 10$-fold greater than those assessed in first-voided and pre-massage midstream urine. Inflammatory leukocytes were counted in expressed prostatic secretions, post-massage urine and semen. In cases where infection by more than one pathogen was detected, microbiological tests were repeated to exclude accidental specimen contamination. Validated Italian versions of the NIH-CPSI and International Prostate Symptom Score (IPSS) tests were used to score the severity of the clinical symptoms (22).

The baseline workup of each candidate to aminoglycoside treatment included audiometric testing and measurement of serum creatinine.

Study outline. After completing clinical and microbiological evaluations at the baseline visit (visit time-point 0, 'V0'), patients were treated with netilmicin $(4.5 \mathrm{mg} / \mathrm{kg}$ body weight, once-daily, intramuscular), combined or not with a $\beta$-lactam antibiotic. In Italy, treatment of prostatitis is an approved indication for aminoglycosides such as netilmicin; hence, administration of these agents was on-label, non-experimental and part of routine practice. Patients remained from time-point V0 to time-point V6 on continuous treatment with the $\alpha$-adrenoceptor blocker alfuzosin (10 mg/day), combined with a supplement containing a Serenoa repens extract (640 mg/day), lycopene (5 mg/day) and selenium (50 mg/day) in a single formulation (Profluss ${ }^{\circledR}$, Konpharma, Rome, Italy) (23). Ketoprofen (160 mg, twice daily) was administered rectally to a group $(n=11)$ of mildly febrile patients until normalization of the temperature was achieved.

At 4 weeks after the end of the antimicrobial treatment all patients were subjected to a complete diagnostic protocol including microbiological and clinical evaluations. This time-point was designated as visit for assessment of pathogen eradication (VERAD). 
At the V6 and V12 follow-up visits ( 6 and 12 months after VERAD, respectively), patients were subjected to complete clinical evaluations.

Microbiological response evaluation. The definitions of Naber et al were used to report the microbiological response to antibacterial therapy: i) Eradication: baseline pathogen was eradicated $\left(<10^{3} \mathrm{CFU} / \mathrm{ml}\right)$; ii) eradication with superinfection: baseline pathogen was eradicated $\left(<10^{3} \mathrm{CFU} / \mathrm{ml}\right)$ with the appearance of a new pathogen $\left(\geq 10^{3} \mathrm{CFU} \mathrm{ml}\right)$; iii) persistence: baseline pathogen was not eradicated $\left(\geq 10^{3} \mathrm{CFU} / \mathrm{ml}\right)$; iv) persistence with superinfection: baseline pathogen was persistent $\left(\geq 10^{3} \mathrm{CFU} / \mathrm{ml}\right)$ with the appearance of a new pathogen $\left(\geq 10^{3} \mathrm{CFU} / \mathrm{ml}\right)(24)$.

Aminoglycoside ototoxicity risk assessment. There were 38 patients who gave written consent for genetic analysis limited to the $1555 \mathrm{~A}>\mathrm{G}, 1494 \mathrm{C}>\mathrm{T}$ and $1556 \mathrm{C}>\mathrm{T}$ mutations in the mitochondrial DNA, which predispose an individual to irreversible sensorineural hearing loss following exposure to aminoglycosides. A specimen of cells was obtained from each patient by gently scraping the inner cheek mucosa with a sterile mucosal brush. Mitochondrial DNA was extracted, using the Quick-DNA ${ }^{\mathrm{TM}}$ Universal kit and the DNA Clean \& Concentration kit (both Zymo Research Corp., Irvine, CA, USA) according to the manufacturer's protocol, and used as a template for the identification of ototoxicity-predisposing mutations by two different techniques: Polymerase chain reaction-restriction fragment length polymorphism (PCR-RFLP) (25) and multiplex PCR (26). The full sequences of the primers, the final concentration of all reagents in the reaction, and the cycling conditions were identical to those described by the developers of the two analytical techniques $(25,26)$. All personnel analyzing the genetic material and the outcome assessor were blinded to the identity of the patients. The genetic material was destroyed immediately following the release of the anonymized results of the mutation analysis. The entire procedure (handling of genetic material and data) was legitimated by the General Authorization No. 8/2014 for the Processing of Genetic Data, of the Italian Data Protection Authority.

Data handling and statistical analysis. To analyze pre- vs. post-therapy paired differences in NIH-CPSI and IPSS scores the Wilcoxon signed-rank test was used; intergroup differences (eradicated vs. persistent cases) were analyzed with the Mann-Whitney-Wilcoxon rank sum test. The measure of central tendency for NIH-CPSI and IPSS ordinal scores was the median, and the interquartile range (IQR) was used to indicate statistical data dispersion. For continuous variables [urinary peak flow rate, serum prostate-specific antigen (PSA) concentration and prostate volume], paired or unpaired t-tests were used to analyze differences between means. An $\alpha$ error of $<5 \%$ was set as the significance level for each comparison. Comparison between paired patient proportions was made using the McNemar's test for correlated proportions.

The VassarStats on-line statistics platform (vassarstats. net) and the JASP software (jasp-stats.org/) were used for the statistical analysis of data. Post-hoc computation of achieved power was performed using the $\mathrm{G}^{*}$ Power 3.1 program (27).

\section{Results}

Clinical presentation of patients and treatment protocols. From the clinical database, the data of 93 patients affected by category II CBP, for whom therapy with aminoglycoside antibiotics was initially considered, were retrieved. The main reasons for aminoglycoside indication were resistance of causative pathogens to fluoroquinolones, intolerance to fluoroquinolones (for example, a history of drug-induced tendonitis or severe gastrointestinal disturbances) or ineffectiveness of previous cycles of therapy with fluoroquinolones (Table I).

Fifteen patients refused treatment or were excluded from therapy following preliminary otolaryngological tests, kidney function evaluations, or careful scrutiny of personal and family history (Table II), and 78 cases (median age, 43 years; IQR, 22 years) initiated treatment with aminoglycosides, combined or not with other antibiotics ( $\beta$-lactams).

Five different antibacterial protocols were adopted. Table III summarizes these protocols, together with the rationale for use of the listed agents. All protocols contained the aminoglycoside netilmicin $(4.5 \mathrm{mg} / \mathrm{kg}$, intramuscular), administered once-daily.

Eight patients were noncompliant with therapy or dropped-out during treatment and were excluded from the present per-protocol efficacy analysis.

There were 11 patients who presented with low-grade pyrexia $\left(<38.3^{\circ} \mathrm{C} / 101^{\circ} \mathrm{F}\right)$, but did not show the array of signs and symptoms typical of acute bacterial prostatitis. In all cases, fever resolved within 72-96 h after the first dose of antibiotic. As prostate massage is contraindicated in febrile patients until normalization of the temperature occurs, the initial diagnosis of prostatitis was based on sonography, clinical signs and symptoms and a midstream urine culture, eventually followed by the '4-glass' test.

Safety of aminoglycoside therapy. Therapy was in general well tolerated. Adverse effects possibly linked to aminoglycoside exposure were reported in two cases. In 1 patient the serum creatinine level increased from $1.1 \mathrm{mg} / \mathrm{dl}$ at time-point $\mathrm{V} 0$ to $2.3 \mathrm{mg} / \mathrm{dl}$ at time-point VERAD. This effect was reversible, and the serum creatinine level dropped to $1.2 \mathrm{mg} / \mathrm{dl}$ 8 weeks later. One patient complained of tinnitus by the end of treatment, and audiometry confirmed hearing impairment, with a notch at $8 \mathrm{KHz}$. The examining otolaryngologist suggested that therapy might have induced the worsening of a pre-existing high-frequency hearing impairment.

Microbiological results. Eradication of the causative pathogen at time-point VERAD was observed in 55 out of 70 per-protocol patients $(78.6 \%)$ and 15 patients exhibited microbiological persistence. No cases of superinfection were reported.

There were 45 and 22 patients that presented with a single-pathogen infection caused by a gram-negative or gram-positive pathogen, respectively, whereas 3 patients had mixed gram-positive/gram-negative infections (Table IV). Escherichia coli and Enterococcus faecalis were the most prevalent pathogens (35 and 19 isolates, respectively). Single-pathogen E.faecalis and E.coli infections were eradicated in 56 and $85 \%$ of cases, respectively (Table IV). Cases 
Table I. Rationale for aminoglycoside therapy.

Adverse effects to first-line agents

Persistence after first-line agents

Adverse effects to first-line agents

Garstine agents
Gastrointestinal
Tendonitis
Resistance of causative pathogens to first-line agents
Patients, $\mathrm{n}$

\begin{tabular}{|c|c|c|c|c|}
\hline Yes & & & & 3 \\
\hline & Yes & & & 11 \\
\hline & & Yes & & 7 \\
\hline & & & Yes & 15 \\
\hline Yes & & Yes & & 2 \\
\hline & Yes & & Yes & 5 \\
\hline & & Yes & Yes & 6 \\
\hline Yes & Yes & Yes & & 6 \\
\hline & Yes & Yes & & 14 \\
\hline & Yes & Yes & Yes & 9 \\
\hline
\end{tabular}

of infection recurrence were not reported in the cohort of 70 treated patients.

Microbiological eradication at time-point VERAD was observed in 10 out of 11 febrile patients (90.91\%), suggesting that aminoglycoside therapy might be an effective therapeutic option in certain complicated cases. Such therapy can usually be managed in an outpatient setting.

Clinical and laboratory findings. The clinical findings of the total patient population at enrollment (V0), at test of eradication (VERAD) and 6 or 12 months thereafter (time-points V6 and V12, respectively) are summarized in Fig. 1 and Table V.

Whereas the symptoms of prostatitis did not improve in the group of patients exhibiting pathogen persistence, the median total NIH-CPSI score, as well as the pain, voiding symptom and quality of life (QoL) subdomain scores of the NIH-CPSI decreased significantly in patients showing microbiological eradication at time-point VERAD, and showed further significant attenuation during follow-up. A caveat is that the statistical power and robustness of unpaired comparisons between groups showing microbiological eradication versus persistence are questionable, as cohorts show considerably different sizes ( $\mathrm{n}=55$ vs. $\mathrm{n}=15$, respectively), and baseline imbalances are present (for example, baseline prostate volume).

NIH-CPSI total score. In patients showing pathogen eradication, the NIH-CPSI total score decreased significantly from a baseline median value of 21 to 14 at time-point VERAD, and to 9 and 8 at time-points V6 and V12, respectively $(\mathrm{P}<0.001$ for all paired comparisons vs. V0, Wilcoxon signed rank test, $\mathrm{n}=55$ ). The differences between VERAD and V6/V12, and between V12 and V6 are also significant ( $\mathrm{P}<0.001$; Wilcoxon, $\mathrm{n}=55)$.

In patients showing persistence of infection, the score at VERAD was not significantly different from baseline values.
A clinically appreciable reduction (28) of $\geq 6$ points of the total score of the NIH-CPSI questionnaire was achieved in 32 out of $55(58 \%)$ patients in which infection was eradicated, and in 4 out of $15(26 \%)$ patients with persistent infection $(\mathrm{P}<0.05$, two-tailed probability associated with the $\mathrm{z}$-ratio, $\mathrm{n}=55$ ).

NIH-CPSI pain domain. In the patients in which eradication of infection was successful, the NIH-CPSI pain score decreased significantly at VERAD, declined further at time-point V6, and remained unchanged at V12 (Fig. 1). In patients showing persistence of infection, the median pain score at VERAD was not significantly different from baseline values.

The disease severity was categorized using the full pain domain score of the NIH-CPSI, according to Wagenlehner's criteria (29). At time-point V0, 27 patients in the eradicated group showed mild pain ( $0-7$ points), 22 patients moderate pain (8-13 points) and 6 severe pain (14-21 points). At the end of therapy 49,6 and 0 patients showed mild, moderate and severe pain, respectively $[\mathrm{P}<0.01$, two-tailed McNemar's test for correlated proportions, dichotomized data (mild vs. moderate/severe), $\mathrm{n}=55$ ].

Voiding signs and symptoms. Voiding symptom scores decreased significantly at VERAD in the eradicated group, and were sustained at time-points V6 and V12 (Fig. 1). This finding was also evident when the results of the IPSS test were analyzed (Table V). In patients showing persistence of infection, the voiding symptom scores at VERAD were not significantly different from baseline values.

The reduction of voiding symptoms was confirmed by assessment of the peak urinary flow and of the bladder voiding efficiency: Mean Qmax increased from $17.93 \mathrm{ml} / \mathrm{sec}$ at $\mathrm{V} 0$ to $18.97 \mathrm{ml} / \mathrm{sec}$ at VERAD, and the percentage bladder voided volume (\%BVV; $89 \%$ of total bladder content at V0) was also significantly increased at VERAD $(98 \%)(\mathrm{P}<0.05$ for 
Table II. Rationale for rejection or contraindication of treatment following initial general indication of aminoglycoside therapy.

Rationale Patients, $\mathrm{n}$

Professional musician

Semiprofessional involvment in music or professional activity requiring fine hearing 5

Referred history of reversible hearing impairment upon treatment with aminoglycosides 2

High frequency $(4,000 \mathrm{~Hz})$ hearing impairment assessed during audiometry performed

prior to aminoglycoside therapy initiation

2

Bilateral hearing impairment in family with matrilinear hearing loss

2

Elevated serum creatinine assessed during previous exposure to amikacin

Unilateral hearing impairment referred during anamnestic interview prior to aminoglycoside

therapy initiation

Referred tinnitus plus recurrent otitis

Table III. Antibacterial protocols administered to CBP patients and rationale for drug selection.

Therapeutic

protocol

Drugs and dosages

Rationale
Netilmicin, intramuscular, $4.5 \mathrm{mg} / \mathrm{kg}$, once daily for 2 weeks. Cefuroxime axetil, oral, $250 \mathrm{mg}$, twice daily for 2 weeks

2 Netilmicin, intramuscular, $4.5 \mathrm{mg} / \mathrm{kg}$, once daily for 2 weeks. Cefoperazone, intramuscular, $1 \mathrm{~g}$, twice daily for 2 weeks

3

Netilmicin, intramuscular, $4.5 \mathrm{mg} / \mathrm{kg}$, once daily for 2 weeks. Piperacillin, 2 g, plus tazobactam, $250 \mathrm{mg}$, intramuscular, once daily for 2 weeks

4 Netilmicin, intramuscular, $4.5 \mathrm{mg} / \mathrm{kg}$, once daily for 2 weeks. Co-amoxiclav $875 \mathrm{mg}+125 \mathrm{mg}$, oral, twice daily for 2 weeks 5

Netilmicin, intramuscular, $4.5 \mathrm{mg} / \mathrm{kg}$, once daily for 2 weeks
Cefuroxime was administered to exploit the synergic activity of combined aminoglycosides and $\beta$-lactams; this 2 nd generation cephalosporin showssufficient prostate penetration (43). Pathogen sensitivity was assessed by antibiogram

Cefoperazone was administered to exploit the synergic activity of combined aminoglycosides and $\beta$-lactams; this 3rd generation cephalosporin shows sufficient prostate penetration (43).

Pathogen sensitivity was assessed by antibiogram

Piperacillin was administered to exploit the synergic activity of combined aminoglycosides and $\beta$-lactams in the presence of resistance to cephalosporins (pathogen sensitivity assessed by antibiogram), or upon cephalosporin contra-indication. Piperacillin shows adequate prostate penetration (44)

Co-amoxiclav can be recovered in the prostate tissue (44), and was administered to exploit the synergic activity of combined aminoglycosides and $\beta$-lactams in the presence of resistance to cephalosporins (pathogen sensitivity assessed by antibiogram), or of contraindication to cephalosporins or piperacillin

Netilmicin was administered as single agent when causative pathogens showed resistance to $\beta$-lactams.

$\mathrm{CBP}$, chronic bacterial prostatitis.

both comparisons, paired, two-tailed t-test, $\mathrm{n}=55$; Table V); these two signs remained sustainedly improved throughout the entire follow-up period.

In patients showing pathogen persistence, a significant increase of the peak urinary flow (from $18.2 \mathrm{ml} / \mathrm{sec}$ at V0 to $19.9 \mathrm{ml} / \mathrm{sec}$ at VERAD) was observed. In this cohort, as well as in the larger cohort of patients in which infection was eradicated, the administration of alfuzosin up to time-point V6 may have acted as a major confounder, and no conclusion can be drawn from data concerning short-term improvement of voiding symptoms. However, Fig. 1 shows that the improvement of voiding symptoms was sustained at the 1-year follow-up time-point (V12), when patients had been off alfuzosin therapy for 6 months.

NIH-CPSI QoL domain. Pain and voiding symptom relief, assessed with the NIH-CPSI test, resulted in significant and sustained attenuation of the impact of the disease on QoL 
Table IV. Microbiological eradication of each pathogen, assessed at time-point VERAD in the per-protocol study cohort of a total of 70 patients.

\begin{tabular}{lccc}
\hline Pathogen & Eradicated, $\mathrm{n}(\%)$ & Persistent, $\mathrm{n}(\%)$ & Total cases \\
\hline Escherichia coli & $29(85.29)$ & $5(14.71)$ & 34 \\
Enterococcus faecalis & $9(56.25)$ & $7(43.75)$ & 16 \\
Staphylococcus aureus & $2(100.00)$ & - & 2 \\
Proteus mirabilis & $2(100.00)$ & $1(33.33)$ \\
Klebsiella oxytoca & $2(66.67)$ & - & 2 \\
Pseudomonas aeruginosa & $1(100.00)$ & $1(100.00)$ \\
Enterobacter cloacae & - & $1(50.00)$ \\
Morganella morganii & $1(50.00)$ & - & \\
Haemophylus spp. & $1(100.00)$ & - & 1 \\
Citrobacter spp. & $1(100.00)$ & - & 2 \\
Streptococcus $\beta$-haemolyticus & $4(100.00)$ & - \\
E. faecalis plus E. coli & $1(100.00)$ & - \\
E. faecalis plus P. aeruginosa & $2(100.00)$ & $15(21.43)$ \\
Total & $55(78.57)$ & 1 \\
\hline
\end{tabular}

VERAD, visit for assessment of pathogen eradication.

Table V. Serum PSA, prostate volume, Qmax, \% voided bladder and IPSS at various time points.

\begin{tabular}{|c|c|c|c|c|}
\hline \multirow[b]{2}{*}{ Variable } & \multicolumn{4}{|c|}{ Study time point } \\
\hline & V0 & VERAD & V6 & V12 \\
\hline \multicolumn{5}{|l|}{ PSA (ng/ml) } \\
\hline Eradicated & $3.32 \pm 2.89$ & $1.89 \pm 1.37^{\mathrm{a}}$ & NA & NA \\
\hline Persistent & $3.31 \pm 3.73$ & $2.29 \pm 1.98^{\mathrm{b}, \mathrm{c}}$ & NA & NA \\
\hline \multicolumn{5}{|c|}{ Prostate volume (ml) } \\
\hline Eradicated & $30.0 \pm 15.44$ & $24.6 \pm 12.17^{\mathrm{a}}$ & NA & NA \\
\hline Persistent & $25.5 \pm 9.21$ & $25.27 \pm 14.50^{\mathrm{c}, \mathrm{d}}$ & NA & NA \\
\hline \multicolumn{5}{|c|}{$\mathrm{Qmax}(\mathrm{ml} / \mathrm{sec})$} \\
\hline Eradicated & $17.93 \pm 7.25$ & $18.97 \pm 5.62^{b}$ & $19.32 \pm 5.48^{b}$ & $18.66 \pm 4.06^{\mathrm{b}}$ \\
\hline Persistent & $18.21 \pm 5.60$ & $19.93 \pm 5.73^{\mathrm{b}, \mathrm{c}}$ & NA & NA \\
\hline \multicolumn{5}{|c|}{$\%$ voided bladder } \\
\hline Eradicated & $88.95 \pm 17.25$ & $97.75 \pm 9.18^{b}$ & $97.90 \pm 8.00^{\mathrm{b}}$ & $96.75 \pm 12.04^{b}$ \\
\hline Persistent & $93.85 \pm 11.37$ & $97.66 \pm 9.03^{\mathrm{c}, \mathrm{d}}$ & NA & NA \\
\hline \multicolumn{5}{|l|}{ IPSS score } \\
\hline Eradicated & $12(9)$ & $8.5(5.5)^{\mathrm{e}}$ & $7(4.25)^{\mathrm{e}}$ & $7(5)^{e}$ \\
\hline Persistent & $10.25(3)$ & $9(3)^{\mathrm{f}, \mathrm{g}}$ & NA & NA \\
\hline
\end{tabular}

Results were measured at baseline (V0), at test of microbiological eradication (VERAD), and at 6 or 12 months follow-up (V6 and V12, respectively). Results are stratified according to the microbiological outcome of therapy (eradicated vs persistent infection). Median values and interquartile ranges (in parentheses) of the IPSS, and mean \pm standard deviations of all other endpoints are presented. Due to the retrospective nature of the study, some follow-up data are missing, as patients showing microbiological persistence were managed with a different, third-level diagnostic and therapeutic protocol. ${ }^{a} \mathrm{P}<0.0001 \mathrm{vs}$. V0, paired t-test; ${ }^{b} \mathrm{P}<0.05 \mathrm{vs}$. V0, paired $\mathrm{t}$-test; ${ }^{\mathrm{c}} \mathrm{P} \geq 0.05$, intergroup comparison (unpaired t-test); ${ }^{\mathrm{d}} \mathrm{P} \geq 0.05$ vs. V0, paired t-test; ${ }^{\mathrm{e}} \mathrm{P}<0.05$ vs. V0, paired comparison (Wilcoxon signed rank test) ; ${ }^{\mathrm{f}} \mathrm{P} \geq 0.05$ vs. V0, paired comparison (Wilcoxon signed rank test); ${ }^{\mathrm{P}} \mathrm{P} \geq 0.05$, intergroup comparison (Mann-Whitney-Wilcoxon rank sum test). VERAD, visit for assessment of pathogen eradication; IPSS, International Prostate Symptom Score; Qmax, urinary peak flow rate; PSA, serum prostate-specific antigen; NA, not assessed.

in patients with eradiation of infection, but not in patients showing persistent infection (Fig. 1).
Sonography and laboratory assessments. A significant reduction of the prostatic volume was identified in patients showing 

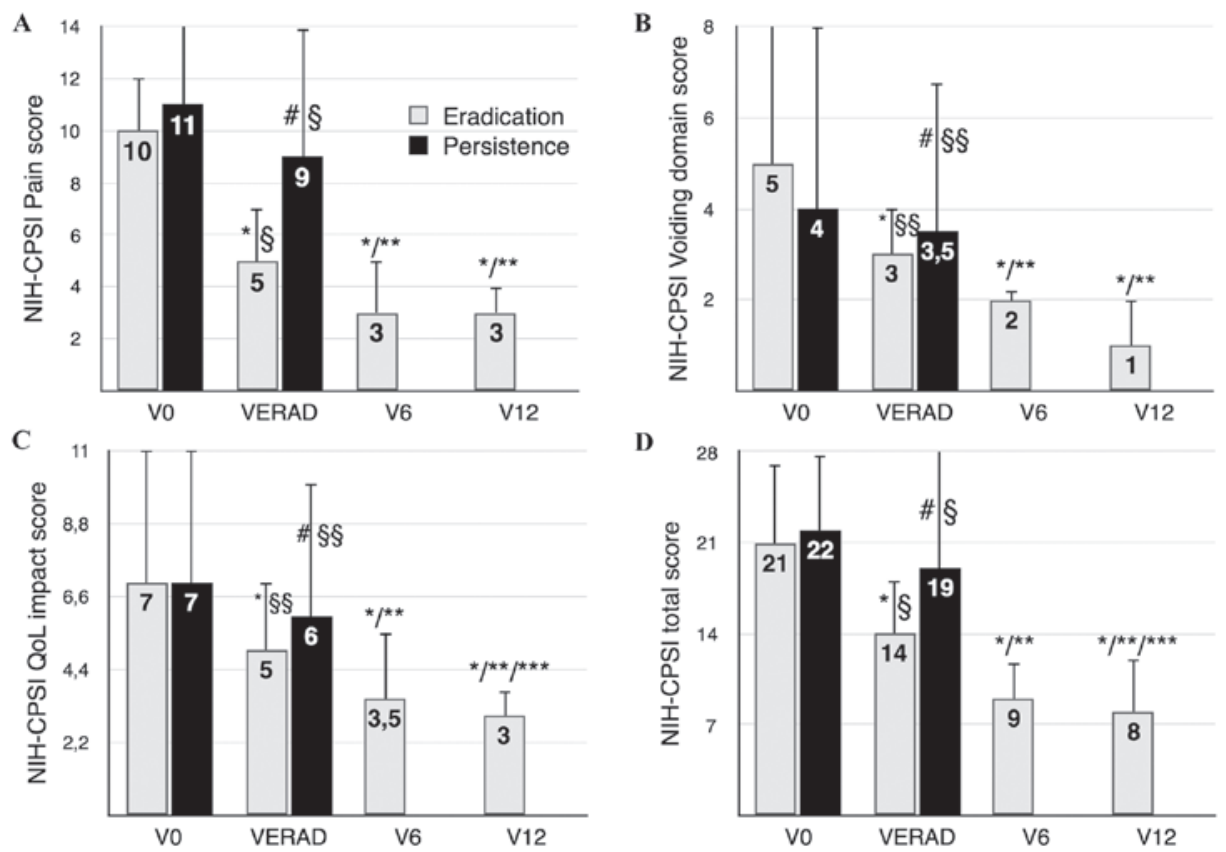

Figure 1. NIH-CPSI scores, assessed at baseline (time-point V0), at test of microbiological eradication (VERAD), and at follow-up (V6 or V12, 6 or 12 months after VERAD, respectively). Results are stratified according to the microbiological outcome of therapy (eradication vs. persistence of infection). Median values of NIH-CPSI scores are indicated, and interquartile range bars are shown. (A) Pain domain, (B) voiding symptoms domain, (C) impact of the disease on patients' QoL and (D) total NIH-CPSI scores. Due to the retrospective nature of the study, the follow-up data of patients showing microbiological persistence are missing, as these patients were routed towards a third-level diagnostic and therapeutic protocol. ${ }^{*} \mathrm{P}<0.001 \mathrm{vs.} \mathrm{V} 0$, paired comparison (Wilcoxon signed rank test). ${ }^{* *} \mathrm{P}<0.001$ vs. VERAD, paired comparison (Wilcoxon signed rank test). ${ }^{* * * * *} \mathrm{P}<0.05$ vs. V6, paired comparison (Wilcoxon signed rank test). ${ }^{*} \mathrm{P} \geq 0.05 \mathrm{vs}$. V0, paired comparison (Wilcoxon signed rank test). ${ }^{\$} \mathrm{P}<0.01$, intergroup comparison at VERAD (eradication vs. infection persistence groups), Mann-Whitney-Wilcoxon rank sum test. ${ }^{8} \mathrm{P} \geq 0.05$, intergroup comparison at VERAD (eradication vs. infection persistence groups), Mann-Whitney-Wilcoxon rank sum test. NIH-CPSI, National Institutes of Health Chronic Prostatitis Symptom Index; VERAD, visit for assessment of pathogen eradication; QoL, quality of life.

pathogen eradication at time-point VERAD (mean difference, $5.4 \mathrm{ml}$ lower; $\mathrm{P}<0.0001$, paired t-test, $\mathrm{n}=55)$, but not in patients not responding to the antibacterial therapy $(25.27 \mathrm{ml}$ at VERAD vs. $25.5 \mathrm{ml}$ at $\mathrm{V0} ; \mathrm{P} \geq 0.05, \mathrm{n}=15$ ).

The mean PSA serum level decreased significantly in the eradicated group (mean difference, $0.23 \mathrm{ng} / \mathrm{ml}$ lower; $\mathrm{P}<0.0001, \mathrm{n}=55$ ). However, PSA also decreased significantly in patients showing pathogen persistence (2.29 at VERAD vs. 3.31 at $\mathrm{V} 0 ; \mathrm{P}<0.05, \mathrm{n}=15)$.

Study power. Post-hoc computation of achieved power of the analyses was based on an $\alpha$ error probability of 0.05 , a calculated correlation between paired datasets equal to 0.7 and a sample size of 55 patients with eradicated infection. The estimated power for the pre- and post-therapy paired comparison of the total score of the NIH-CPSI test was $>0.9$. Sensitivity analysis, given a sample size of 55, a normal distribution (two-tailed), an $\alpha$ error probability of 0.05 , and an imputed power of 0.99 , resulted in a required effect size of 1.007 (the effect size for the above comparison calculated in the present study was 1.2).

\section{Discussion}

Few drugs are able to reach the prostatic ducts or acini via the systemic circulation, due to the existence of a blood-prostate barrier activity preventing the distribution of a number of chemicals, including antibacterial agents, to the various cells and regions within the gland (30).
Goto et al authored one of the few studies investigating the distribution of aminoglycosides and other four different antibacterials to prostatic fluid/secretions (31). It was shown that the aminoglycoside amikacin (200 mg, intramuscular) reached the highest concentrations in prostatic fluids $(3.2 \mu \mathrm{g} / \mathrm{ml})$, compared with $\beta$-lactams (piperacillin, $0.3 \mu \mathrm{g} / \mathrm{ml}$ ), tetracyclines (minocycline, $0.62 \mu \mathrm{g} / \mathrm{ml}$ ) or fluoroquinolones (ofloxacin, $1.3 \mu \mathrm{g} / \mathrm{ml}$ ), although the latter showed the highest prostatic fluid-to-serum ratio (ofloxacin: 0.9; amikacin: 0.25) (31). Notably, patients enrolled in the study by Goto et al were not affected by acute prostatitis, known to facilitate the distribution of any drug to the prostate (31). Thus, positive evidence is available about the distribution of certain aminoglycosides to the sites of chronic infection in the prostate.

Cure of CBP achieved with aminoglycosides was reported as early as 1976 and 1991 by Pfau and Sacks in isolated cases or a small series of patients treated with parenteral kanamycin ( $1 \mathrm{~g}$ twice daily for 3 days, and $500 \mathrm{mg}$ twice daily for 11 days; $\mathrm{n}=13)$ or streptomicin ( $1 \mathrm{~g} /$ day for 12 days; 1 case) $(32,33)$, but to the best of our knowledge the overall evidence in this respect is scant.

Within the limits of an observational study, the data in the present study show that microbiological eradication and sustained clinical symptom remission was achieved in $79 \%$ of patients (or $71 \%$, if a intent-to-treat setting is considered, including 8 noncompliant/dropped-out patients) subjected to a course of treatment with an aminoglycoside, administered alone or in combination with a $\beta$-lactam antibiotic. Notably, 
similar pathogen eradication rates, ranging between 70 and $80 \%$, have been reported in the past in CBP patients treated with various fluoroquinolones $(19,24,34,35)$. In summary, the results of the present study suggest that aminoglycosides may be administered to selected CBP patients, provided that candidates to therapy are at low risk for the serious adverse effects of these agents.

Therapy with aminoglycosides exposes patients to the risk of hearing impairment. In particular, the carriers of the $1555 \mathrm{~A}>\mathrm{G}$ point-mutation within the mitochondrial DNA locus of the $12 \mathrm{~S}$ ribosomal RNA develop profound and irreversible non-syndromic sensorineural hearing loss even after short-term exposure to aminoglycosides (36). One in $520(0.19 \%)$ European children, 6 in 865 Chinese newborns $(0.7 \%)$, and 1 in 500 adults of European descent $(0.2 \%)$ are estimated to be carriers of this mutation, and are at risk for such severe toxic effects (37-39). Other important ototoxicity-predisposing mutations have been discovered, for example the $1494 \mathrm{C}>\mathrm{T}$ and $1556 \mathrm{C}>\mathrm{T}$ substitutions in the same $12 \mathrm{~S}$ rRNA $(40,41)$. In our routine clinical practice, consenting patient candidates for aminoglycoside therapy are screened for a small panel of mutations predisposing to severe, irreversible hearing loss. This preliminary analysis can be performed in few hours prior to the first dose of the antibiotic, and allows safer administration of aminoglycosides. However, a negative pharmacogenetic examination result does not exempt patients from less severe forms of hearing impairment, possibly caused by reactive oxygen species. Administration of aspirin has been shown to partly protect patients from these forms of ototoxicity (42).

In conclusion, administration of aminoglycosides eradicated causative pathogens in $79 \%$ of patients and significantly decreased the symptoms of the disease, as assessed with the NIH-CPSI and IPSS questionnaires. The impact of CBP on the QoL of patients was also significantly attenuated. Therapy was also effective in significantly decreasing the prostate volume, probably by acting on inflammatory edema. The therapy was in general well tolerated, and genetic testing for mitochondrial mutations predisposing to sensorineural deafness allowed safer administration of the antibiotic in a considerable fraction of patients. Thus, the data presented in this paper indicate that aminoglycosides may be an interesting option for the treatment of CBP in specific, carefully selected cases. These findings should be confirmed and further validated in a randomized-controlled setting.

\section{References}

1. Dennis LK, Lynch CF and Torner JC: Epidemiologic association between prostatitis and prostate cancer. Urology 60: 78-83, 2002.

2. Roberts RO, Bergstralh EJ, Bass SE, Lieber MM and Jacobsen SJ: Prostatitis as a risk factor for prostate cancer. Epidemiology 15 : 93-99, 2004.

3. Rybicki BA, Kryvenko ON, Wang Y, Jankowski M, Trudeau S, Chitale DA, Gupta NS, Rundle A and Tang D: Racial differences in the relationship between clinical prostatitis, presence of inflammation in benign prostate and subsequent risk of prostate cancer. Prostate Cancer Prostatic Dis 19: 145-150, 2016.

4. Boehm K, Valdivieso R, Meskawi M, Larcher A, Schiffmann J, Sun M, Graefen M, Saad F, Parent MÉ and Karakiewicz PI: Prostatitis, other genitourinary infections and prostate cancer: Results from a population-based case-control study. World J Urol 34: 425-430, 2016.
5. Jiang J, Li J, Yunxia Z, Zhu H, Liu J and Pumill C: The role of prostatitis in prostate cancer: Meta-analysis. PLoS One 8: e85179, 2013.

6. Chung SD, Keller JJ and Lin HC: A case-control study of chronic prostatitis/chronic pelvic pain syndrome and colorectal cancer. BJU Int 110: 550-554, 2012.

7. Cheng I, Witte JS, Jacobsen SJ, Haque R, Quinn VP, Quesenberry CP, Caan BJ and Van Den Eeden SK: Prostatitis, sexually transmitted diseases, and prostate cancer: The California Men's Health Study. PLoS One 5: e8736, 2010.

8. Krieger JN, Nyberg L Jr and Nickel JC: NIH consensus definition and classification of prostatitis. JAMA 282: 236-237, 1999.

9. Perletti G, Marras E, Wagenlehner FM and Magri V: Antimicrobial therapy for chronic bacterial prostatitis. Cochrane Database Syst Rev 8: CD009071, 2013.

10. Noel GJ, Natarajan J, Chien S, Hunt TL, Goodman DB and Abels R: Effects of three fluoroquinolones on QT interval in healthy adults after single doses. Clin Pharmacol Ther 73: 292-303, 2003

11. Arabyat RM, Raisch DW, McKoy JM and Bennett CL: Fluoroquinolone-associated tendon-rupture: A summary of reports in the Food and Drug Administration's adverse event reporting system. Expert Opin Drug Saf 14: 1653-1660, 2015.

12. Dalhoff A: Global fluoroquinolone resistance epidemiology and implictions for clinical use. Interdiscip Perspect Infect Dis 2012: 976273, 2012.

13. Sarma JB, Marshall B, Cleeve V, Tate D, Oswald T and Woolfrey S: Effects of fluoroquinolone restriction (from 2007 to 2012) on resistance in Enterobacteriaceae: Interrupted time-series analysis. J Hosp Infect 91: 68-73, 2015.

14. Borgmann S, Jakobiak T, Gruber H, Schröder H and Sagel U: Ciprofloxacin treatment of urinary infections results in increased resistance of urinary E. coli to ciprofloxacin and co-trimoxazole. Pol J Microbiol 58: 371-373, 2009.

15. Brede CM and Shoskes DA: The etiology and management of acute prostatitis. Nat Rev Urol 8: 207-212, 2011.

16. Etienne M, Chavanet P, Sibert L, Michel F, Levesque H, Lorcerie B, Doucet J, Pfitzenmeyer P and Caron F: Acute bacterial prostatitis: Heterogeneity in diagnostic criteria and management. Retrospective multicentric analysis of 37 patients diagnosed with acute prostatitis. BMC Infect Dis 8: 12, 2008.

17. Hanus PM and Danziger LH: Treatment of chronic bacterial prostatitis. Clin Pharm 3: 49-55, 1984.

18. von Elm E, Altman DG, Egger M, Pocock SJ, Gøtzsche PC and Vandenbroucke JP; STROBE Initiative: The Strengthening the Reporting of Observational Studies in Epidemiology (STROBE) statement: Guidelines for reporting observational studies. Lancet 370: 1453-1457, 2007.

19. Magri V, Montanari E, Skkerk V, Markotić A, Marras E, Restelli A, Naber KG and Perletti G: Fluoroquinolone-macrolide combination therapy for chronic bacterial prostatitis: Retrospective analysis of pathogen eradication rates, inflammatory findings and sexual dysfunction. Asian J Androl 13: 819-827, 2011.

20. Stamey TA: Prostatitis. J R Soc Med 74: 22-40, 1981.

21. Magri V, Wagenlehner FM, Montanari E, Marras E, Orlandi V, Restelli A, Torresani E, Naber KG and Perletti G: Semen analysis in chronic bacterial prostatitis: Diagnostic and therapeutic implications. Asian J Androl 11: 461-477, 2009.

22. Giubilei G, Mondaini N, Crisci A, Raugei A, Lombardi G, Travaglini F, Del Popolo G and Bartoletti R: The Italian version of the National Institutes of Health Chronic Prostatitis Symptom Index. Eur Urol 47: 805-811, 2005

23. Morgia G, Mucciardi G, Galì A, Madonia M, Marchese F, Di Benedetto A, Romano G, Bonvissuto G, Castelli T, Macchione L, et al: Treatment of chronic prostatitis/chronic pelvic pain syndrome category IIIA with Serenoa repens plus selenium and lycopene (Profluss) versus S. repens alone: An Italian randomized multicenter-controlled study. Urol Int 84: 400-406, 2010.

24. Naber KG; European Lomefloxacin Prostatitis Study Group: Lomefloxacin versus ciprofloxacin in the treatment of chronic bacterial prostatitis. Int J Antimicrob Agents 20: 18-27, 2002

25. Casano RA, Bykhovskaya Y, Johnson DF, Hamon M, Torricelli F, Bigozzi M and Fischel-Ghodsian N: Hearing loss due to the mitochondrial A1555G mutation in Italian families. Am J Med Genet 79: 388-391, 1998.

26. Scrimshaw BJ, Faed JM, Tate WP and Yun K: Rapid identification of an A1555G mutation in human mitochondrial DNA implicated in aminoglycoside-induced ototoxicity. J Hum Genet 44: 388-390, 1999. 
27. Faul F, Erdfelder E, Lang AG and Buchner A: G*Power 3 A flexible statistical power analysis program for the social, behavioral, and biomedical sciences. Behav Res Methods 39: 175-191, 2007.

28. Propert KJ, McNaughton-Collins M, Leiby BE, O'Leary MP, Kusek JW and Litwin MS; Chronic Prostatitis Collaborative Research Network: A prospective study of symptoms and quality of life in men with chronic prostatitis/chronic pelvic pain syndrome: The National Institutes of Health Chronic Prostatitis Cohort study. J Urol 175: 619-623, 2006.

29. Wagenlehner FM, van Till JW, Magri V, Perletti G, Houbiers JG, Weidner W and Nickel JC: National Institutes of Health Chronic Prostatitis Symptom Index (NIH-CPSI) symptom evaluation in multinational cohorts of patients with chronic prostatitis/chronic pelvic pain syndrome. Eur Urol 63: 953-959, 2013.

30. Shang Y and Cui D and Yi S: Opening tight junctions may be key to opening the blood-prostate barrier. Med Sci Monit 20: 2504-2507, 2014.

31. Goto T, Makinose S, Ohi Y, Yamauchi D, Kayajima T, Nagayama K and Hayami H: Diffusion of piperacillin, cefotiam, minocycline, amikacin and ofloxacin into the prostate. Int J Urol 5: 243-246, 1998.

32. Pfau A: The treatment of chronic bacterial prostatitis. Infection 19 (Suppl 3): S160-S164, 1991

33. Pfau A and Sacks T: Chronic bacterial prostatitis: New therapeutic aspects. Br J Urol 48: 245-253, 1976.

34. Bundrick W, Heron SP, Ray P, Schiff WM, Tennenberg AM Wiesinger BA, Wright PA, Wu SC, Zadeikis N and Kahn JB: Levofloxacin versus ciprofloxacin in the treatment of chronic bacterial prostatitis: A randomized double-blind multicenter study. Urology 62: 537-541, 2003.

35. Magri V, Trinchieri A, Pozzi G, Restelli A, Garlaschi MC, Torresani E, Zirpoli P, Marras E and Perletti G: Efficacy of repeated cycles of combination therapy for the eradication of infecting organisms in chronic bacterial prostatitis. Int J Antimicrob Agents 29: 549-556, 2007.
36. Fischel-Ghodsian N, Prezant TR, Chaltraw WE, Wendt KA, Nelson RA, Arnos KS and Falk RE: Mitochondrial gene mutation is a significant predisposing factor in aminoglycoside ototoxicity. Am J Otolaryngol 18: 173-178, 1997.

37. Bitner-Glindzicz M, Pembrey M, Duncan A, Heron J, Ring SM, Hall A and Rahman S: Prevalence of mitochondrial 1555A-->G mutation in European children. N Engl J Med 360: 640-642, 2009.

38. Vandebona H, Mitchell P, Manwaring N, Griffiths K, Gopinath B, Wang JJ and Sue CM: Prevalence of mitochondrial 1555A $>$ G mutation in adults of European descent. N Engl J Med 360: 642-644, 2009.

39. Chen G, Wang X and Fu S: Prevalence of A1555G mitochondrial mutation in Chinese newborns and the correlation with neonatal hearing screening. Int J Pediatr Otorhinolaryngol 75: 532-534, 2011.

40. Zhao H, Li R, Wang Q, Yan Q, Deng JH, Han D, Bai Y, Young WY and Guan MX: Maternally inherited aminoglycoside-induced and nonsyndromic deafness is associated with the novel C1494T mutation in the mitochondrial $12 \mathrm{~S}$ rRNA gene in a large Chinese family. Am J Hum Genet 74: 139-152, 2004.

41. Tanimoto H, Nishio H, Matsuo M and Nibu K: A novel mitochondrial mutation, $1556 \mathrm{C}-->\mathrm{T}$, in a Japanese patient with streptomycin-induced tinnitus. Acta Otolaryngol 124: 258-261, 2004.

42. Sha SH, Qiu JH and Schacht J: Aspirin to prevent gentamicin-induced hearing loss. N Engl J Med 354: 1856-1857, 2006.

43. Charalabopoulos K, Karachalios G, Baltogiannis D, Charalabopoulos A, Giannakopoulos X and Sofikitis N: Penetration of antimicrobial agents into the prostate. Chemotherapy 49: 269-279, 2003.

44. Kobayashi I, Ikawa K, Nakamura K, Nishikawa G, Kajikawa K, Yoshizawa T, Watanabe M, Kato Y, Zennami K, Kanao K, et al: Penetration of piperacillin-tazobactam into human prostate tissue and dosing considerations for prostatitis based on site-specific pharmacokinetics and pharmacodynamics. J Infect Chemother 21: 575-580, 2015. 